Ann. Biol. anim. Bioch. Biophys., I963, 3 (2), I03-II7.

\title{
ETUDE DE L'INGESTION SPONTANÉE D'ÉLÉMENTS ÉNERGÉTIQUES ET DE PROTÉINES CHEZ LE RAT EN CROISSANCE PAR LA METHODE DU LIBRE CHOIX
}

\author{
Y. HENRY et A. RÉRAT \\ avec la collaboration technique de Marie-Claire Therox, Françoise Hollier et Yvonne Daniel \\ Station de Recherches sur l'Élevage des Porcs, \\ Centre national de Recherches zootechniques, Jouy-en-Josas (Seine-et-Oise)
}

\section{SOMMAIRE}

L'ingestion spontanée des éléments énergétiques et azotés chez le Rat en croissance a été étudiée en distribuant simultanément, dans deux mangeoires séparées et pendant 8 semaines, un régime protéique ad libitum et un régime protéiprive offert également à volonté. A cet effet, trois lots ont été constitués, recevant l'une des trois sources azotées suivantes : farine de poisson, tourteau d'arachide, gluten de blé.

D'après les résultats obtenus, il apparaît que le Rat est incapable d'équilibrer le taux azoté de son régime au niveau de son besoin. Par contre, il existe une relation étroite entre la consommation spontanée d'énergie et le poids. Ceci confirme l'hypothèse de l'aịustement de la consommation de nourriture sur le besoin énergétique.

\section{INTRODUCTION}

Il est connu, depuis les premiers travaux d'Osborne et MExdei, (IgI7) que la consommation de nourriture et, par conséquent, d'énergie chez l'animal en croissance, est fonction de la composition du régime, et plus précisément de sa teneur en protéines et de la nature de ces dernières. Réciproquement, il a été montré que l'utilisation des matières azotées pour la croissance dépend de la quantité d'énergie consommée par l'animal (MUNRo, I95I). Autrement dit, l'intensité de la croissance et l'efficacité du régime sont liées à un équilibre convenable entre l'énergie et les matières azotées. Mais cet équilibre n'est pas obligatoirement réalisé dans les condi- 
tions habituelles d'alimentation. Ainsi CALET et al. (I96r) ont démontré que le poulet, recevant simultanément dans deux mangeoires séparées les protéines et le reste de la ration, ingère spontanément une quantité d'énergie variable selon la nature de la protéine. Lorsqu'il s'agit d'une protéine de haute qualité, telle que la farine de poisson, cette consommation spontanée d'énergie est plus élevée en régime séparé qu'en régime complet, à quantité d'azote ingérée égale, et entraîne une meilleure utilisation de la protéine. Dans tous les cas, le poulet se fixe un rapport azote-énergie optimum selon la nature de la source azotée; il en est de même pour le rat (ABRAham et al., I96I ; HENRY et Rerat, I962 ; RERAt, HeNRy et JaCquot, I962).

On peut alors se demander si l'animal est capable par lui-même d'ajuster ses consommations d'énergie et d'azote au niveau de ses besoins lorsqu'on lui offre à volonté à la fois l'énergie et l'azote, et non plus l'énergie à volonté pour une quantité d'azote donnée. Dès I915, Evvard avait constaté que le porc en libre consommation est en mesure de composer un régime qui lui assure une croissance aussi bonne, sinon meilleure, qu'un régime imposé. De leur côté, OSBORNE et MENDEL (I9I8) observaient un comportement identique du rat. Cependant, les avis sont assez partagés sur le point de savoir si l'ingestion spontanée constitue un facteur de régulation du besoin. Mitcheli et MENDEL (I92I), Richter et al.(I938) pensent que le rat opère un choix judicieux parmi les aliments qui lui sont offerts, même lorsqu'il s'agit de substances simples (protéines, graisses, glucides, sels minéraux). De même LASSITER et al. (I955) ont montré que le porc qui reçoit à volonté du maïs et un concentré azoté ajuste le taux azoté de son régime à un niveau correspondant à son besoin de croissance. D'autres auteurs, par contre, concluent à l'inaptitude de l'animal à équilibrer son régime en libre choix; AsHKEXASY-LELU (I95I) constate que des rats ainsi nourris réalisent la même croissance qu'en régime imposé, mais au prix d'un gaspillage très important de protéines. A l'inverse Kox (I93I) rapporte que des rats en libre choix, consomment une quantité insuffisante de protéines et dépérissent.

Afin d'étudier les modalités d'ajustement de l'ingestion de nourriture en libre choix nous avons entrepris une expérience sur des rats en leur distribuant simt1ltanément deux repas séparés, un repas azoté ad libitum et un repas non azoté également administré à volonté.

\section{MATÉRIEI, ET MÉTHODES}

Des rats blancs de souche II istar, pris au sevrage, sont placés en cages individuelles et reçoivent pendant 8 jours, ad libitum, un régime préexpérimental à base de caséine (tabl. I). A l'issue de cette période, ils sont répartis en 3 lots homogènes de 9 animaux ( 5 mâles et 4 femelles), d'après leurs poids et gain de poids.

L'expérience consiste à distribuer à chaque animal pendant 8 semaines, dans deux mangeoires séparées, ad libitum, d'un côté un régime protidique contenant une source azotée de nature variable (farine de poisson, tourteau d'arachide, guten de blé), de l'autre un régime protéiprive composé de sucre, huile, cellulose, minéraux et vitamines.

Les trois sources azotées utilisées sont d'origine connue : farine de poisson de Norvège, tourteau d'arachide d'extraction, gluten de blé d'Australie, et renferment respectivement 79,6, 59,8 et $8 \mathrm{I}, 5$ p. Ioo de matières azotées $(\mathrm{N} \times 6,25)$ par rapport à la matière sèche. D'après les dosages effectués par PION (I963) la composition de ces protéines en amino acides indispensables (tabl. 2) est très différente. Leur classe chimique, calculée selon la méthode de MrTchell et Block (I946), permet de les hiérarchiser par ordre d'efficacité prévisible décroissante (poisson : 63 ; arachide :34; gluten : 22). 
TABLEAU I

Composition du régime préexpérimental

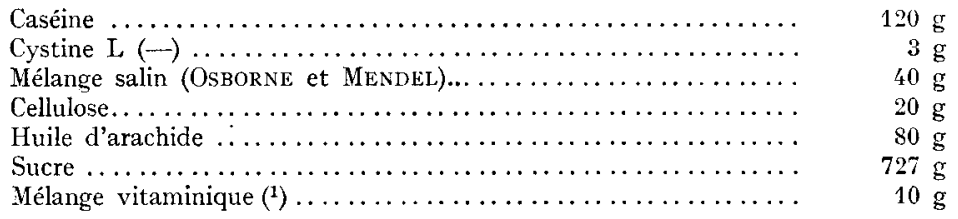

(1) Composition du mélange vitaminique :

\begin{tabular}{|c|c|}
\hline Thiamine (chlorhydrate) & 8 \\
\hline Acide nicotinique $\ldots \ldots \ldots \ldots \ldots \ldots \ldots \ldots \ldots$ & 30 \\
\hline$\ldots \ldots \ldots \ldots \ldots \ldots \ldots \ldots$ & 8 \\
\hline$\ldots \ldots \ldots \ldots \ldots \ldots \ldots$ & 15 \\
\hline Pantothénate de calcium $\ldots \ldots \ldots \ldots \ldots \ldots \ldots$ & 30 \\
\hline 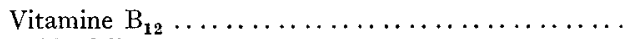 & 0,03 \\
\hline 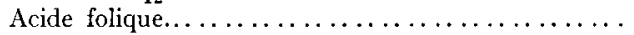 & 1 \\
\hline Inositol $\ldots \ldots \ldots \ldots \ldots \ldots \ldots \ldots \ldots \ldots \ldots \ldots \ldots \ldots \ldots \ldots \ldots$ & 500 \\
\hline Acide paraaminobenzoique............... & 500 \\
\hline 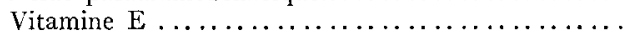 & 30 \\
\hline$\ldots \ldots \ldots \ldots \ldots \ldots \ldots$ & 5 \\
\hline 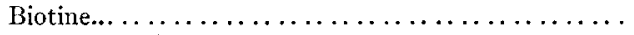 & 0,2 \\
\hline Amidon Q. S. P..... & 10 \\
\hline
\end{tabular}

Les vitamines $A$ et $D$ sont administrées dans l'huile d'arachide sous forme commerciale ( $4 \mathrm{ml}$ de "vitadone "Byla pour $1000 \mathrm{ml}$ d'huile).

La choline est ajoutée au régime à raison de $5 \mathrm{ml}$ d'une solution à 20 p. 100 par kilogramme de régime.

TABLEAU 2

Composition des protéines en acides aminés (PION, 1963)

\begin{tabular}{|c|c|c|c|}
\hline & $\begin{array}{c}\text { Farine de poisson } \\
\text { de Norvège }\end{array}$ & $\begin{array}{l}\text { Tourteau } \\
\text { d'arachide }\end{array}$ & $\begin{array}{l}\text { Gluten de } \\
\text { blé }\end{array}$ \\
\hline $\begin{array}{c}\text { Matière azotées totales }(\mathrm{N} \times 6,25) \text { p. } 100 \text { matière } \\
\text { sèche } \ldots \ldots \ldots \ldots \ldots \ldots \ldots \ldots \ldots \ldots \ldots \ldots \ldots \ldots\end{array}$ & 79,6 & 59,8 & 81,5 \\
\hline \multicolumn{4}{|l|}{ Quantité pour $16 \mathrm{~g}$ d'azote $\left({ }^{1}\right)$} \\
\hline Arginine $\ldots \ldots \ldots \ldots \ldots \ldots \ldots \ldots \ldots$ & 7,3 & 11,4 & 3,6 \\
\hline Cystine $\ldots \ldots \ldots \ldots \ldots \ldots \ldots \ldots \ldots$ & 1,1 & 1,5 & - \\
\hline Histidine $\ldots \ldots \ldots \ldots \ldots \ldots \ldots \ldots$ & 2,4 & 2,2 & 2,1 \\
\hline Isoleucine $\ldots \ldots \ldots \ldots \ldots \ldots \ldots$ & 5,4 & 3,2 & 4,2 \\
\hline Leucine $\ldots \ldots \ldots \ldots \ldots \ldots \ldots$ & 8,2 & 6,0 & 7,0 \\
\hline Lysine $\ldots \ldots \ldots \ldots \ldots \ldots \ldots$ & 7,7 & 3,4 & 1,6 \\
\hline Méthionine $\ldots \ldots \ldots \ldots \ldots \ldots \ldots \ldots$ & 2,6 & 0,5 & 1,4 \\
\hline 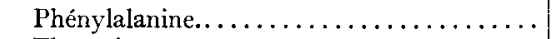 & 4,2 & 4,1 & 5,4 \\
\hline 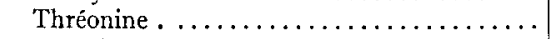 & 4,9 & 2,6 & 2,6 \\
\hline Tyrosine $\ldots \ldots \ldots \ldots \ldots \ldots \ldots \ldots$ & 3,7 & 3,7 & 3,5 \\
\hline Valine $\ldots \ldots \ldots \ldots \ldots \ldots \ldots \ldots \ldots$ & 6,3 & 4,2 & 4,4 \\
\hline Phénylalanine + tyrosine...... & 7,9 & 7,8 & 8,9 \\
\hline Méthionine + cystine...$\ldots \ldots \ldots$ & 3,7 & 2,0 & 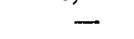 \\
\hline
\end{tabular}

(1) Dosages par chromatographie (Moore et STEIN). 
Le régime protidique est constitué par parties égales de la source azotée et du régime protéiprive et renferme entre $3^{6}$ et $3^{8} \mathrm{p}$. Ioo de protéines. La composition de ces régimes est présentée dans le tableau 3 .

TABLEAU 3

Composition des régimes

\begin{tabular}{|c|c|c|c|c|}
\hline \multirow[b]{2}{*}{ Source azotée } & \multicolumn{3}{|c|}{ Régime protéique } & \multirow{2}{*}{$\begin{array}{c}\text { Régime } \\
\text { protéiprive }\end{array}$} \\
\hline & $\begin{array}{c}\text { Farine de } \\
\text { poisson }\end{array}$ & $\begin{array}{c}\text { Tourteau } \\
\text { d'arachide }\end{array}$ & $\begin{array}{c}\text { Gluten de } \\
\text { blé }\end{array}$ & \\
\hline 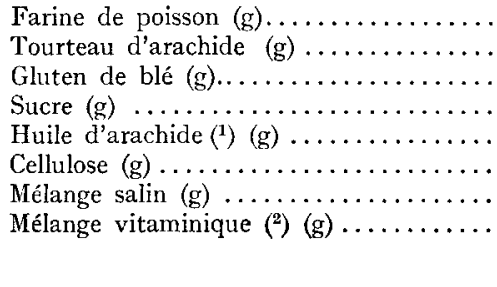 & $\begin{array}{r}1000 \\
- \\
- \\
835 \\
90 \\
20 \\
45 \\
10 \\
2000\end{array}$ & $\begin{array}{r}\overline{1360} \\
\overline{475} \\
90 \\
20 \\
45 \\
10 \\
2000\end{array}$ & $\begin{array}{r}- \\
- \\
940 \\
895 \\
90 \\
20 \\
45 \\
10 \\
2000\end{array}$ & $\begin{array}{r}- \\
- \\
835 \\
90 \\
20 \\
45 \\
10 \\
1000\end{array}$ \\
\hline
\end{tabular}

(I) Les vitamines $\mathrm{A}$ et $\mathrm{D}$ sont incorporées à l'huile d'arachide ( $4 \mathrm{ml}$ de " vitadone "Byla pour $1000 \mathrm{ml}$ d'huile).

(2) La biotine et la choline sont introduites à part sous forme de solution à raison de $5 \mathrm{ml}$ d'une solution à 20 p. 100 de choline et $5 \mathrm{ml}$ d'une solution à 0,040 p. 1000 de biotine par kg d'aliment. Les mélanges salin et vitaminique sont ceux utilisés dans le régime préexpérimental (tabl. 1).

La consommation de nourriture est enregistrée quotidiennement. La teneur énergétique des régimes est évaluée d'après les coefficients d'ATwATER et BRYANT (I903) obtenus à l'aide de la bombe calorimétrique. Ces données servent de base pour l'appréciation de la consommation spontanée d'énergie exprimée en calories totales.

Les animaux sont pesés deux fois par semaine. A la fin de l'expérience, ils sont sacrifiés et conservés en chambre froide à — $20^{\circ} \mathrm{C}$. Les carcasses entières ainsi congelées sont broyées séparément dans un hachoir électrique Bauknecht: ; après deux passages dans cet appareil, le broyat est homogénéisé manuellement à l'aide d'un masticateur. On procède à l'analyse de la matière sèche, des cendres et de l'azote sur des parties aliquotes de ce broyat ; les lipides sont obtenus par différence (matière sèche - cendres - $\mathrm{N} \times 6,25$ ). Un test comparatif nous a permis de vérifier que cette méthode d'estimation des lipides par différence donne des résultats très voisins de ceux obtenus par une technique de dosage au chloroforme suivant la méthode de BLIGH et DyER (I959); les pourcentages de lipides trouvés sur so échantillons très différents du point de vue de la teneur en lipides ont été : Io, 72 (par différence) et Io,4I (par dosage), soit un écart de 3 p. Ioo seulement par excès.

La rétention azotée est calculée par différence entre l'azote final dosé dans la carcasse et l'azote initial estimé. L'équation de régression qui permet d'estimer l'azote initial a été obtenue à partir des résultats d'analyse corporelle de 52 animaux témoins abattus à l'issue de la période préexpérimentale; exprimée sous forme logarithmique, elle s'écrit :

$$
\log \mathrm{N}=\mathrm{I}, 4765+0,96 \mathrm{r} \log \mathrm{P}
$$

$\mathrm{N}$ étant la quantité d'azote contenue dans la carcasse en $g$, et $\mathbf{P}$ le poids en $\mathrm{g}$ (RERAT et al., résultats non publiés).

De même que les calories ingérées, les calories retenues dans la carcasse sont obtenues en utilisant les coefficients d'ATWATER et BRYANT.

Les calculs statistiques ont été effectués suivant la méthode d'analyse de variance et, pour la comparaison des moyennes, le test de Keuls-Newman, (SNEdEcor, 1956) a été utilisé. 


\section{RÉSULTATS}

Résultats de croissance et de consommation après 8 semaines

Les résultats généraux après les 8 semaines d'expérience sont rapportés dans le tableau 4 pour les animaux mâles et dans le tableau 5 pour les animaux femelles. Il convient tout d'abord de noter, pour tous les critères, une différence considérable entre ces deux catégories d'animaux (tab1.6). Quoi qu'il en soit, mâles et femelles répondent de la même façon vis-à-vis de la nature de la source azotée, puisqu'il n'existe aucune interaction significative entre cette dernière et le sexe ; c'est pourquoi la comparaison des protéines entre elles a été faite sur l'ensemble des animaux.

TABLEAU 4

Résultats générautu à 56 jours (animaux mâles)

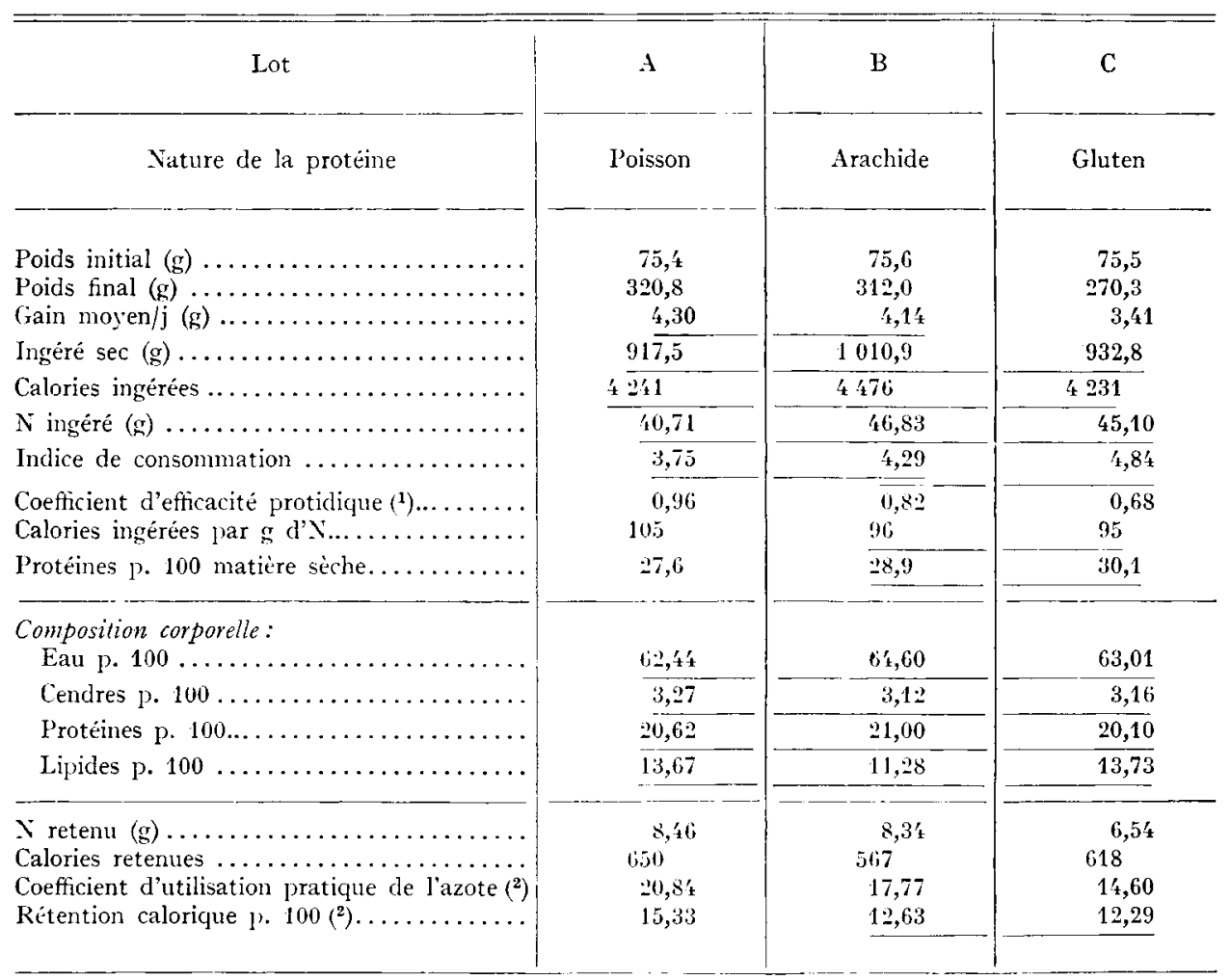

(1) Coefficient d'efticacité protidique : $\frac{\text { yain de poids }(\mathrm{g})}{\text { protéunes }\left(\mathrm{N} \times \frac{\mathrm{b}, 25) \text { ingérées }(\mathrm{g})}{6}\right.}$.

$\left({ }^{2}\right)$ Coefficient d'utilisation pratique de l'azote $: \frac{N \text { corporel final }(g)-N \text { corporel initial }(g)}{N \text { ingéré }(g)}$.

(3) Rétention calorique $\left[\right.$. $100: \frac{\text { calories retenues }}{\text { calories ingérées }} \times 100$.

Les moyennes non réunies par une ligne sont différentes au seuil 0,05 ; celles qui sont soulignées ensemble ne sont pas significativement différentes (test de KEULS-NEWMAN).

Annales de Biologie animale. - I963. 
TABI,EAU 5

Résultats généraux à 56 jours (animaux femelles)

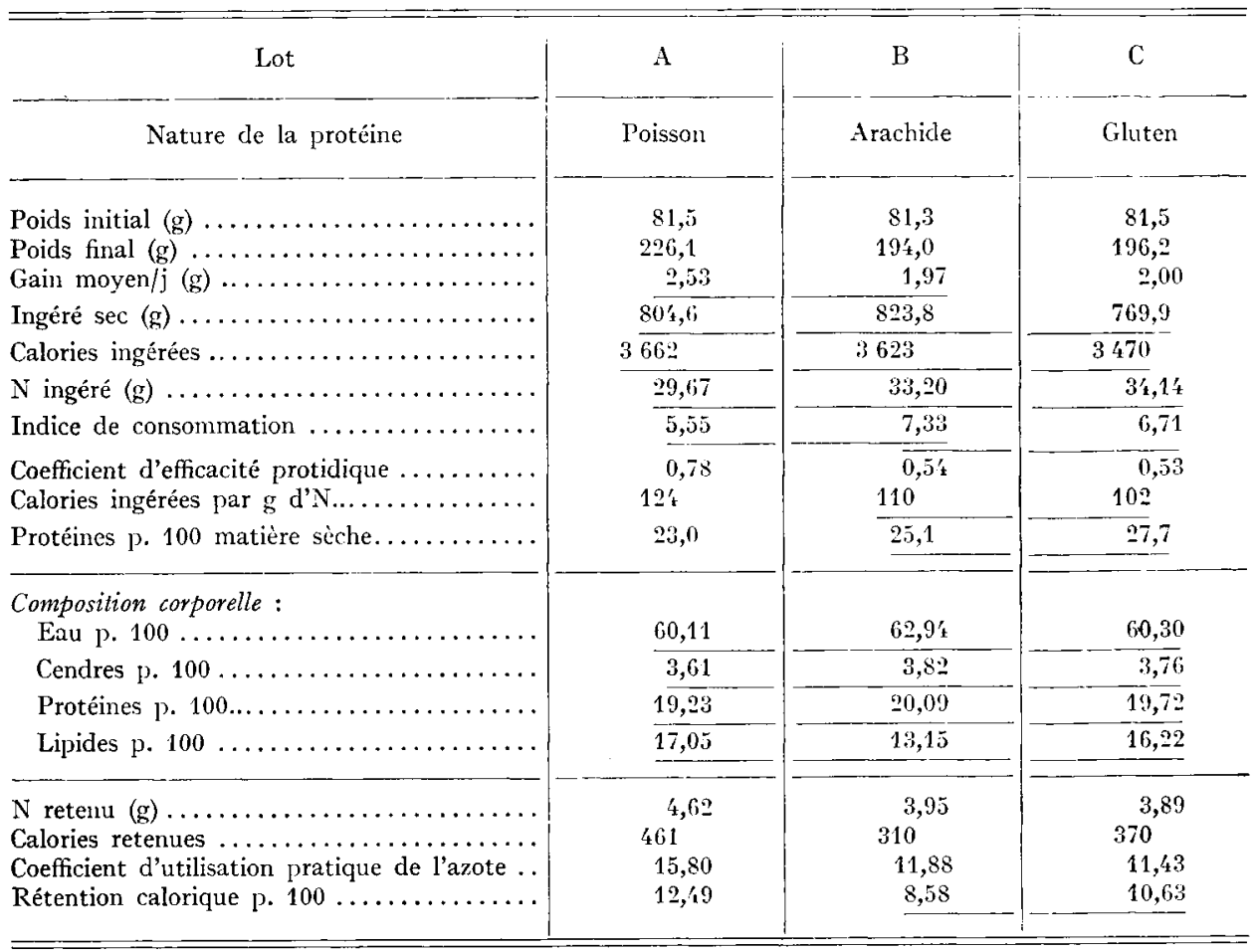

\section{TABLEAU 6}

Interprétation statistique des résultats à 56 jours (infuence du sexe)

\begin{tabular}{|c|c|c|}
\hline & $\begin{array}{l}\text { Carré moyen de l'effet sexe } \\
\text { (DL }=1)\end{array}$ & $\begin{array}{l}\text { Erreur } \\
(\mathrm{DL}=21)\end{array}$ \\
\hline 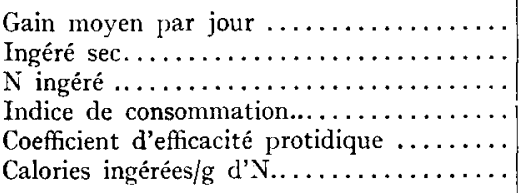 & $\begin{array}{r}21,1109 * * \\
158758 * * \\
932,8087 * * \\
33,9965 * * \\
0,2574 * * \\
12,29,45 * *\end{array}$ & $\begin{array}{l}0,1445 \\
3595 \\
21,4156 \\
0,1795 \\
0,0038 \\
46,94\end{array}$ \\
\hline 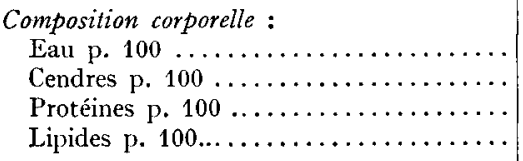 & $\begin{array}{r}33,2568^{*} \\
1,9947^{* *} \\
5,3083^{* *} \\
4 \mathbf{t}, 6337^{*}\end{array}$ & $\begin{array}{l}4,7099 \\
0,0514 \\
0,5416 \\
8,1871\end{array}$ \\
\hline $\begin{array}{l}\text { Coefficient d'utilisation pratique de l'azote } \\
\text { Rétention calorique p. } 100 \ldots \ldots \ldots \ldots \ldots \ldots\end{array}$ & $\begin{array}{r}1: 7,308: 4 * * \\
54,1563^{* *}\end{array}$ & $\begin{array}{l}2,2941 \\
2,3118\end{array}$ \\
\hline
\end{tabular}

* Seuil de signification : 0,05 .

** Seuil de signification : 0,01 . 
Le gain moyen journalier pendant 8 semaines est d'autant plus élevé que la protéine est de meilleure qualité ; ainsi les animaux recevant de la farine de poisson ont réalisé une croissance plus rapide que ceux recevant du tourteatı d'arachide, lesquels sont à leur tour supérieurs aux animaux consommant du gluten de blé. Cependant les lots poisson et arachide ne sont pas significativement différents ; les courbes de croissance correspondantes sont d'ailleurs très voisines (fig. I).

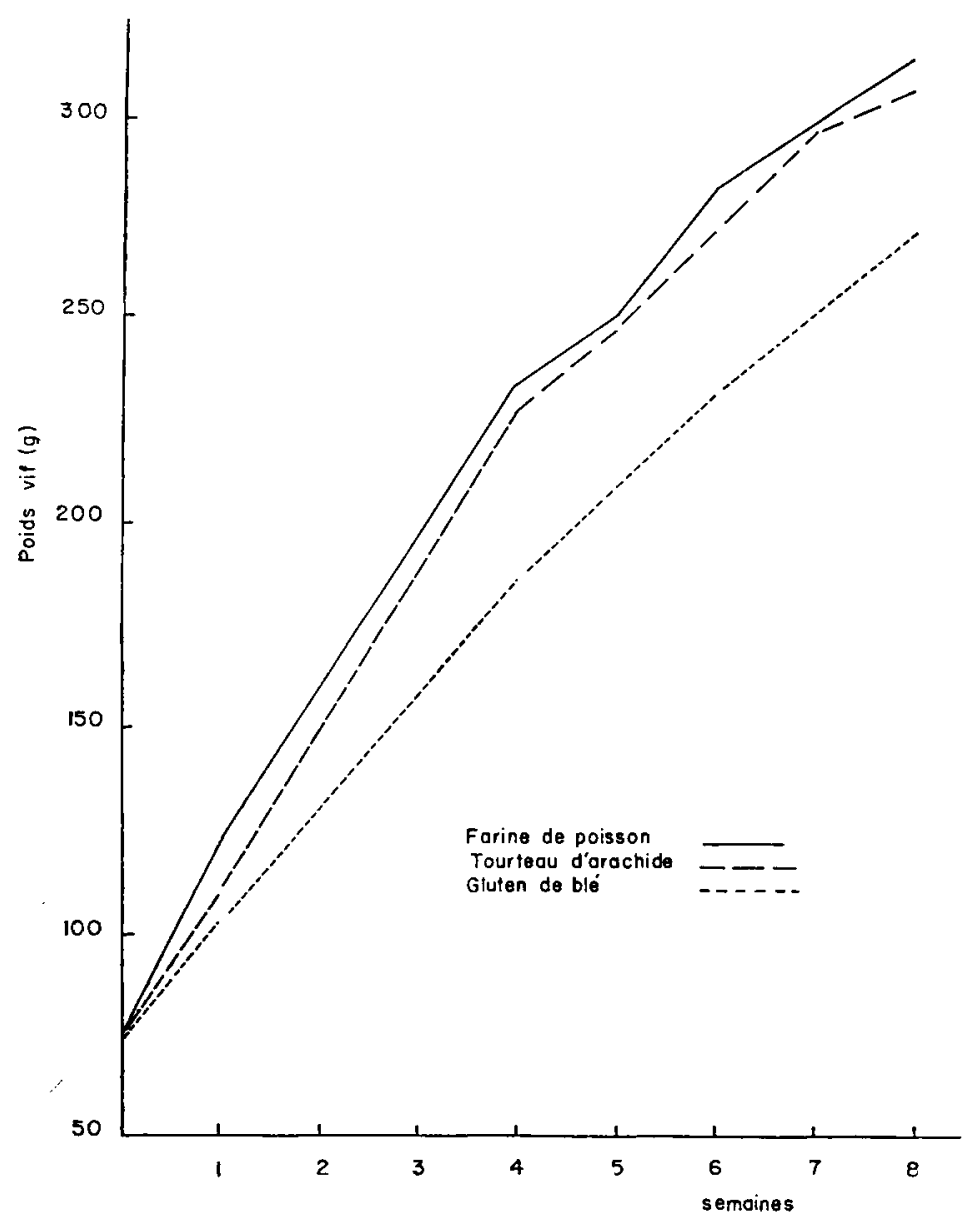

FIG. I. - Courbes de croissance des animaux máles.

L,es consommations de matière sèche et d'azote ne sont pas différentes d'une source azotée à l'autre. Il semble toutefois que les animaux nourris de protéines de qualité médiocre (arachide, gluten) ont tendance à ingérer plus d'azote que ceux nourris de protéines de haute qualité (farine de poisson).

En ce qui concerne l'indice de consommation, l'évolution est la même que pour le gain moyen journalier ; le lot poisson est supérieur au lot arachide, lesquel est supérieur à son tour au lot gluten. Cependant, seuls les lots poisson et gluten sont significativement différents l'un de l'autre. 
Les valeurs obtenues pour le coefficient d'efficacité protidique se classent généralement dans le même ordre que celles obtenues pour le gain moyen journalier, mais sont toutes différentes. Il en est de même du coefficient d'utilisation pratique de l'azote et du pourcentage de rétention calorique. Pour ce dernier critère, il n'existe pas de différence significative entre les lots arachide et gluten.

D'autre part, les rats recevant de la farine de poisson ingèrent plus de calories par gramme d'azote que ceux recevant du tourteau d'arachide ou du gluten; il en résulte un pourcentage plus faible de protéines par rapport à la matière sèche. Dans les trois lots, 1'ingestion de protéines s'est équilibrée à environ $30 \mathrm{p}$. Ioo de 1'ingestion de matière sèche.

TABIEAU 7

Évolution des critères au cours de la croissance (moyennes des animaux mâles)

\begin{tabular}{|c|c|c|c|c|}
\hline \multirow{2}{*}{ Critère } & \multicolumn{4}{|c|}{ Quinzaines } \\
\hline & 1 & 2 & 3 & 4 \\
\hline \multicolumn{5}{|l|}{ Gain moyen journalier $(g)$} \\
\hline $\begin{array}{l}\text { Poisson } \ldots \ldots \ldots \ldots \ldots \ldots \ldots \ldots \ldots \ldots \ldots \ldots \\
\text { Arachide } . \ldots \ldots \ldots \ldots \ldots \ldots \ldots \ldots \ldots \ldots \ldots \\
\text { Gluten } \ldots \ldots \ldots \ldots \ldots \ldots \ldots \ldots\end{array}$ & $\begin{array}{l}5,78 \\
4,8 ! \\
3,76\end{array}$ & $\begin{array}{l}\{, 99 \\
5,38 \\
3,66\end{array}$ & $\begin{array}{l}3,81 \\
3,69 \\
3,28\end{array}$ & $\begin{array}{l}2,53 \\
2,62 \\
2,95\end{array}$ \\
\hline \multicolumn{5}{|l|}{ Calories ingérées/j } \\
\hline 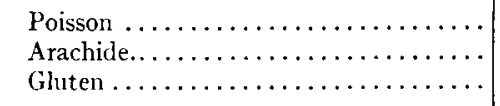 & $\begin{array}{l}61,9 \\
60,0 \\
64,3\end{array}$ & $\begin{array}{l}74,9 \\
80,7 \\
73,7\end{array}$ & $\begin{array}{l}78,5 \\
82,5 \\
77,2\end{array}$ & $\begin{array}{l}83,1 \\
91,7 \\
86,6\end{array}$ \\
\hline \multicolumn{5}{|l|}{$N$ ingérélj (mg) } \\
\hline $\begin{array}{l}\text { Poisson } \ldots \ldots \ldots \ldots \ldots \ldots \ldots \ldots \ldots \ldots \ldots \\
\text { Arachide...... } \\
\text { Gluten } \ldots \ldots \ldots \ldots \ldots \ldots \ldots \ldots \ldots \ldots \ldots \ldots \ldots\end{array}$ & $\begin{array}{l}57 t_{4} \\
628 \\
6 \prime 1\end{array}$ & $\begin{array}{l}7 \% 9 \\
868 \\
80 *\end{array}$ & $\begin{array}{l}768 \\
875 \\
813\end{array}$ & $\begin{array}{l}760 \\
928 \\
888\end{array}$ \\
\hline \multicolumn{5}{|l|}{ Indice de consonmation } \\
\hline $\begin{array}{l}\text { Poisson } \ldots \ldots \ldots \ldots \ldots \ldots \ldots \ldots \ldots \ldots \ldots \\
\text { Arachide... } \ldots \ldots \ldots \ldots \ldots \ldots \ldots \ldots \ldots \\
\text { Gluten } \ldots \ldots \ldots \ldots \ldots \ldots \ldots\end{array}$ & $\begin{array}{l}2,36 \\
2,82 \\
3,53\end{array}$ & $\begin{array}{l}3,25 \\
3,45 \\
4,46\end{array}$ & $\begin{array}{l}4,48 \\
5,23 \\
5,39\end{array}$ & $\begin{array}{l}7,44 \\
8,12 \\
7,21\end{array}$ \\
\hline \multicolumn{5}{|l|}{ Efficacité protidique } \\
\hline 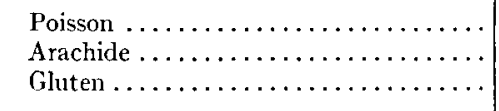 & $\begin{array}{l}1,61 \\
1,23 \\
0,91\end{array}$ & $\begin{array}{l}1,07 \\
0,98 \\
0,73\end{array}$ & $\begin{array}{l}0,79 \\
0,67 \\
0,62\end{array}$ & $\begin{array}{l}0,53 \\
0,45 \\
0,52\end{array}$ \\
\hline \multicolumn{5}{|l|}{ Calories ingérées par $g d^{\prime} N$} \\
\hline 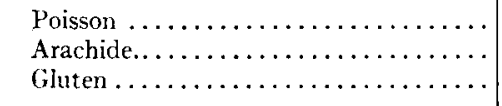 & $\begin{array}{r}108 \\
95 \\
94\end{array}$ & $\begin{array}{r}100 \\
93 \\
92\end{array}$ & $\begin{array}{r}102 \\
9 / 4 \\
93\end{array}$ & $\begin{array}{r}110 \\
99 \\
100\end{array}$ \\
\hline 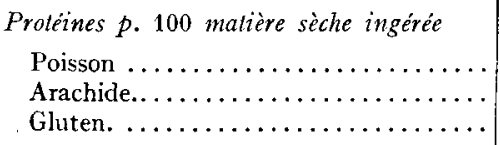 & $\begin{array}{l}26,8 \\
29,1 \\
30,2\end{array}$ & $\begin{array}{l}29,0 \\
29,8 \\
30,9\end{array}$ & $\begin{array}{l}28,3 \\
29,4 \\
31,0\end{array}$ & $\begin{array}{l}26,4 \\
27,9 \\
28,8\end{array}$ \\
\hline
\end{tabular}




\section{Évolution des critères au cours de la croissance}

Afin de suivre le comportement du rat en libre consommation, énergétique et azotée, pendant les 8 semaines d'expérience, nous avons rapporté dans le tableau 7 , pour les animaux mâles, l'évolution des critères de croissance et de consommation par périodes de I4 jours; l'analyse statistique des résultats est détaillée dans le tableau 8 .

TABLEAU 8

Evolution des crilères en fonction du temps - Interprétation statistique des résultats

\begin{tabular}{|c|c|c|c|c|}
\hline & $\mathrm{DL}$ & Poisson & Arachide & Gluten \\
\hline & & & carré moyen & \\
\hline \multicolumn{5}{|l|}{ Gain moyen journalier } \\
\hline Terme linéaire .............. & 1 & $5841,54 * *$ & $3415,23 * *$ & 384,55 \\
\hline Terme quadratique $\ldots \ldots \ldots \ldots$ & 1 & 57,46 & $640,71 *$ & 11,70 \\
\hline Terme cubique $\ldots \ldots \ldots \ldots \ldots$ & 1 & 4,80 & 400,80 & 4,51 \\
\hline Erreur...................... & 16 & 73,85 & 115,60 & 103,03 \\
\hline \multicolumn{5}{|l|}{$\begin{array}{l}\text { Protéines ingérées par quizaine } \\
\text { Périodes. }\end{array}$} \\
\hline 1 versus $2-3-4 \ldots \ldots \ldots \ldots \ldots$ & 1 & $974,45 * *$ & $1986,05^{* *}$ & $1208,71 *$ \\
\hline 2 versus $4 \ldots \ldots \ldots \ldots \ldots \ldots$ & 1 & 2,30 & $68,6^{\prime} t$ & 131,77 \\
\hline 3 versus $2-4 \ldots \ldots \ldots \ldots \ldots \ldots$ & 1 & 3,89 & 13,33 & 0,18 \\
\hline Erreur..................... & 16 & 14,02 & 35,30 & 195,11 \\
\hline \multicolumn{5}{|l|}{ Calories ingérées par quinzaine: } \\
\hline Terme linéaire......$\ldots \ldots$ & 1 & $1130,98 * *$ & $1767,36 * *$ & $1234,12 * *$ \\
\hline Terme quadratique..$\ldots \ldots \ldots$ & 1 & $86,94 *$ & 65,62 & 0 \\
\hline Terme cubique $\ldots \ldots \ldots \ldots \ldots$ & 1 & 26,11 & 124,99 & 34,93 \\
\hline Erreur.................. & 16 & 17,59 & 31,60 & 90,76 \\
\hline
\end{tabular}

Calories ingérées par g d'azote:

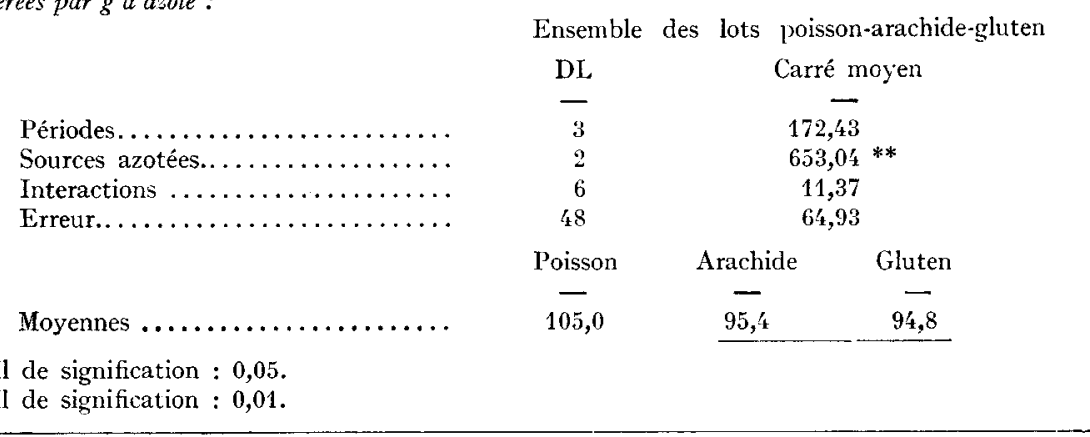

* Seuil de signification : 0,05 .

** Seuil de signification : 0,01 .

Le gain moyen journalier est maximum dès la première quinzaine, dans les lots poisson et gluten, et diminue régulièrement ensuite, tandis que dans le lot arachide il n'atteint son maximum qu'au cours de la deuxième quinzaine. Il convient de noter qu'il s'agissait d'animaux pesant au départ entre 70 et $80 \mathrm{~g}$, c'est ce qui explique qu'ils ont atteint dès le début leur croissance la plus rapide. 
La consommation d'azote croît d'une manière significative entre la première et la deuxième quinzaine pour les 3 sources azotées, puis se maintient à un niveau constant. Par contre, l'ingéré calorique suit une évolution régulière en fonction du temps : cette évolution est linéaire dans le cas de 1'arachide et du gluten, et curvilinéaire dans le cas de la farine de poisson. La différence entre poisson d'une part, et arachide et gluten d'autre part, semblerait indiquer que les rats recevant du poisson ont tendance, en fin de croissance, à limiter leur ingestion de calories ; on peut émettre l'hypothèse que ceci est dû au fait qu'ils sont plus lourds. C'est pourquoi l'évolution de l'ingéré calorique a été étudiée, non plus en fonction du temps, mais en fonction du poids de l'animal.

\section{Relation entre la consommation spontanée d'énergie et le poids}

A la suite des observations précédentes, la consommation journalière de calories par quinzaine a été rapportée, pour chaque animal, au poids moyen pondéré pendant

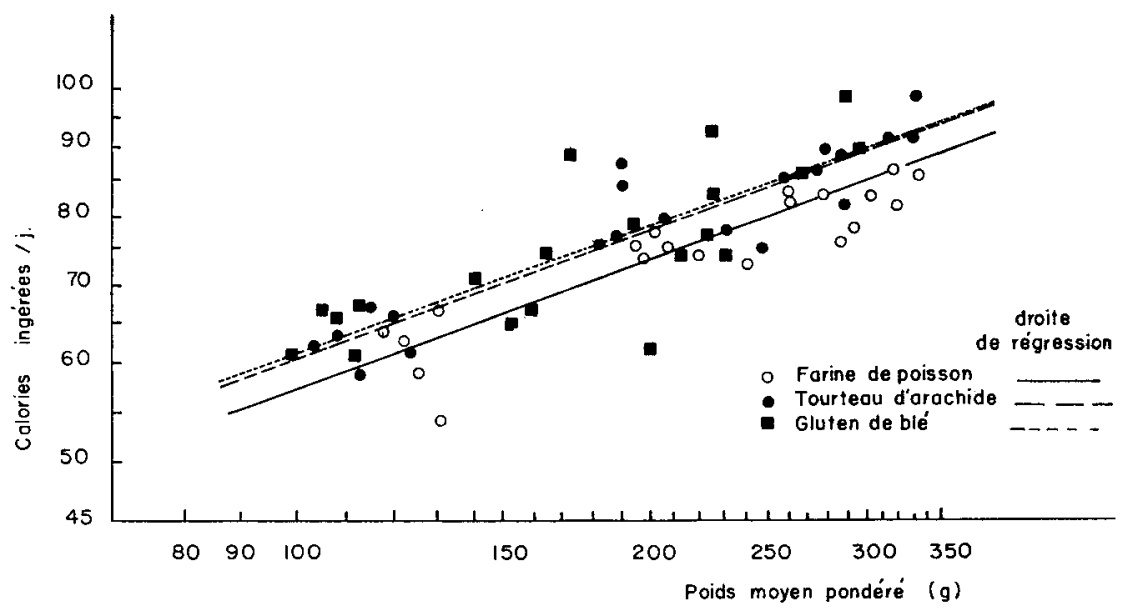

FIG. 2. - Évolution de l'ingéré calorique moyen journalier en fonction du poids moyen pondéré par quinzaine (en coordonnées logarithmiques).

la même période. Le poids moyen pondéré a été choisi comme devant traduire une évolution plus dynamique de la croissance que le poids moyen ; étant représenté par la moyenne de deux poids moyens hebdomadaires successifs, il est donné par la formule :

$$
\mathrm{P}=\mathrm{I} / 2\left[\frac{\left(\mathrm{P}_{1}+\mathrm{P}_{2}\right)}{2}+\frac{\left(\mathrm{P}_{2}+\mathrm{P}_{3}\right)}{2}\right]=\frac{\mathrm{P}_{1}+2 \mathrm{P}_{2}+\mathrm{P}_{3}}{4}
$$

$\mathrm{P}_{1}, \mathrm{P}_{2}$ et $\mathrm{P}_{3}$ étant respectivement les poids au début, au milieu et à la fin de la quinzaine. D'autre part, les calculs ont été effectués sur 2 semaines, plutôt que sur une semaine en raison des variations individuelles de consommation, parfois importantes d'une semaine sur l'autre.

Suivant une méthode déjà exposée dans une précédente publication (HENRY et RERAT, I962), nous avons étudié pour chaque lot, après transformation logarithmique des données, la régression de l'ingéré calorique moyen par animal et par 
quinzaine sur le poids moyen pondéré pendant la période. Une série de droites de régression (fig. 2), dont les équations sont rapportées dans le tableau 9, ont été ainsi obtenues. Ces équations sont de la forme $\log \mathrm{I}=\log a+b \cdot \log \mathrm{P}, \mathrm{I}$ représentant les calories ingérées par jour, $\mathrm{P}$ le poids moyen pondéré en $\mathrm{g}, a$ et $b$ des constantes

TABLEAU 9

Relation entre l'ingéré calorique moyen journalier $(I)$ et le poids moyen pondéré par quinzaine ( $P$

\begin{tabular}{|c|c|c|c|c|c|c|}
\hline Lot & $\begin{array}{c}\text { Nature de la } \\
\text { protéine }\end{array}$ & $\begin{array}{c}\text { Nombre } \\
\text { de } \\
\text { données }\end{array}$ & $\begin{array}{l}\text { Equation de } \\
\text { régression } \log I=\end{array}$ & $\begin{array}{l}\text { Coefficient } \\
\text { de ccrré- } \\
\text { lation }(r)\end{array}$ & \begin{tabular}{|c|} 
Intervalle \\
de confiance \\
de la pente \\
(seuil 0,05 )
\end{tabular} & $\begin{array}{l}\text { Signification } \\
\text { de la régres- } \\
\text { sion (valeur } \\
\text { de } t \text { ) }\end{array}$ \\
\hline$A(\delta)$ & Farine de possion & 20 & $1,10785+0,328 \log P^{1}$ & 0,901 & $\therefore=0,078$ & $8,86 * * *$ \\
\hline $\mathrm{B}\left(\sigma^{*}\right)$ & Tourteau d'arachide & $\because 0$ & $1,08731+0,35: \log \mathrm{P}$ & 0,888 & $\therefore 0,090$ & $8,19 * * *$ \\
\hline$C\left(0^{*}\right)$ & Gluten de blé & 20 & $1,10713+0,343 \log \mathrm{P}$ & 0,759 & 0,145 & $\{, 97 * * *$ \\
\hline Total ot & $\begin{array}{l}\text { Poisson-arachide- } \\
\text { gluten }\end{array}$ & (ii) & $|1,137339+0,325 \log \mathrm{P}|$ & 0,811 & $t=0,062$ & $10,48 * * *$ \\
\hline Total q & $\begin{array}{l}\text { Poisson-arachide- } \\
\text { gluten }\end{array}$ & 47 & $1,06935+0,335 \log \mathrm{l}^{1}$ & 0,800 & $\pm 0,075$ & $9,05^{* * *}$ \\
\hline
\end{tabular}

Seuil de signification : 0,001.

caractéristiques de chaque lot. Cette relation peut encore s'écrire sous la forme exponentielle : $\mathrm{I}=a \cdot \mathrm{P}^{b}$, soit pour l'ensemble des animaux mâles, $\mathrm{I}=\mathrm{I} 3,72 \mathrm{P} \mathbf{0}, 32$.

I)ans les calculs précédents, nous avons considéré séparément les mâles et les femelles. On peut constater que dans l'intervalle de poids considéré (70 à $350 \mathrm{~g}$ ), la consommation spontanée d'énergie chez le rat en croissance est proportionnelle au poids vif élevé à une même puissance (entre 0,32 et 0,35 ), quelle que soit la nature de la source azotée utilisée, et que mâles et femelles se comportent de façon identique. La régression linéaire est dans tous les cas hautement significative et les pentes des droites de régression ne sont pas significativement différentes, d'après les valeurs des intervalles de confiance.

\section{Composition corporelle}

Comme le montrent les tableaux 4 et 5 , la composition corporelle est la même pour les trois lots. Ici encore on peut noter une influence prépondérante du sexe, les mâles étant plus maigres que les femelles (tabl. 6). 


\section{DISCUSSION}

La première constatation qui se dégage des résultats précédents est que le rat est incapable d'ajuster sa consommation d'azote sur son besoin de croissance. En effet, la quantité d'azote ingérée atteint très vite un maximum et se maintient à ce niveau, alors que le gain de poids, et par conséquent le dépôt azoté, diminue régulièrement avec l'âge. Non seulement il n'y a pas d'ajustement dans le temps, mais encore le rat ne semble tenir aucun compte de la qualité de la protéine pour équilibrer son besoin. Ainsi, dans le cas de la farine de poisson, il constitue un régime à 28 p. Ioo de protéines, alors que des expériences poursuivies parallèlement au laboratoire en régime mixte ont montré que la vitesse de croissance est maximum au taux de 16 p. Ioo; d'autre part, le coefficient d'utilisation pratique de l'azote est de $2 x \mathrm{p}$. roo seulement, tandis que la valeur maximum observée est de $40 \mathrm{p}$. IoO (RERAT et al., résultats non publiés). Dans le cas du tourteau d'arachide, nous avons pu constater de la même façon que la croissance est maximum au taux de $24 \mathrm{p}$. Ioo de protéines dans la ration (résultats non publiés). Là encore le rat en libre consommation azotée ingère un excès de protéines, le taux fixé étant de 29 p. Ioo. Par contre, dans le cas d'une protéine de mauvaise qualité comme le gluten de blé, le taux d'azote que l'on peut calculer à partir de l'ingestion spontanée est insuffisant pour assurer la couverture du besoin azoté du rat en croissance. D'après nos propres observations (non publiées), le taux optimum provoquant la meilleure croissance est d'environ $60 \mathrm{p}$. Ioo correspondant à $45 \mathrm{p}$. Ioo de protéines. Il n'est pas exclu cependant que, dans le cas présent, la concentration du régime protidique en gluten ait été insuffisante pour que le rat puisse librement ingérer une plus grande proportion d'azote. Malgré cette réserve, il est intéressant de constater que le taux de protéines que le rat se fixe en libre choix oscille invariablement autour de $30 \mathrm{p}$. Ioo quelles que soient la nature de la protéine et sa valeur biologique. Mais, même à ce niveau, les protéines de poisson se révèlent supérieures aux protéines d'arachide et de gluten, du point de vue de leur utilisation pour la croissance. D'autres auteurs ont constaté également que le rat est incapable de réaliser par lui-même l'équilibre azoté de son régime, soit qu'il ingère une quantité d'azote insuffisante (KON, I93I) ou excessive par rapport à son besoin (ASHKENASY-LELU, I95 I).

Alors que l'ingestion d'azote est indépendante de l'activité métabolique de l'animal, la consommation d'énergie par contre paraît obéir à une loi générale qui fait intervenir le poids, lui-même fonction du niveau de croissance. Quelle que soit la nature de la source azotée, la consommation de calories est proportionnelle au poids élevé à une puissance fixe $(0,32$ à 0,35$)$ dans un intervalle de poids compris entre 70 et $350 \mathrm{~g}$. Dans une précédente publication (HENRY et RERAT, I962), nous avions rapporté une relation comparable lorsqu'on fait varier à la fois la quantité d'azote distribuée et la nature de la source azotée ; la puissance du poids était alors voisine de 0,5 dans un intervalle de poids compris entre 60 et $\mathrm{I} 50 \mathrm{~g}$. Or, selon BroDy (r945), le métabolisme total du rat en croissance est fonction d'une puissance diminuée du poids lorsque ce dernier augmente; cet exposant passe de $0,8-0,9$ pour des rats de moins de Ioo $\mathrm{g}$, à moins de 0,4 au-dessus de roo $\mathrm{g}$, suivant que l'on se trouve de part et d'autre du point d'inflexion de la courbe de croissance. En 
d'autres termes, la valeur de cet exposant est fonction de l'impulsion de croissance de 1'animal. Par ailleurs, HeGsTed et HaffenrefFer (I949) ont montré que 1'ingestion de calories chez des rats recevant différentes protéines à taux variable en régime complet est proportionnelle au poids élevé à la puissance 0,88 , lorsque ce dernier est compris entre 40 et roo g. D'après ces travaux, il apparaît que la consommation d'énergie et la dépense de métabolisme constituent des fonctions exponentielles du poids, l'exposant étant le même dans un même intervalle de poids. Les résultats que nous avons obtenus, tant dans l'expérience présente que dans celle ci-dessus mentionnée, sont en accord avec les conclusions des auteurs précédents. En définitive, on peut en déduire que la quantité d'énergie ingérée spontanément par le rat est en relation directe avec la dépense de métabolisme, ce qui confirme l'hypothèse de l'ajustement de la consommation d'énergie formulée par RUBNER (Ig02) et vérifiée par CowgrL, (I928).

TABLEAU IO

Comparaison de régimes mixtes et séparés assurant le même niveau de croissance (Durée : 56 jours)

\begin{tabular}{|c|c|c|}
\hline Nature de la protéine & Poisson & Poisson \\
\hline Rëgime & $\begin{array}{c}\text { mixte ad libitum } \\
\text { (16 p. } 100 \text { de protéines) }\end{array}$ & $\begin{array}{l}\text { séparé (N ad libitum } \\
\text { énergie ad libitum) }\end{array}$ \\
\hline Poids initial $(g) \ldots \ldots \ldots \ldots \ldots \ldots \ldots$ & 63,4 & 75,4 \\
\hline Poids final $(\mathrm{g}) \ldots \ldots \ldots \ldots \ldots \ldots \ldots$ & 327,1 & 320,8 \\
\hline Gain moyen $/ j(g) \ldots \ldots \ldots \ldots \ldots \ldots$ & 4,71 & 4,30 \\
\hline 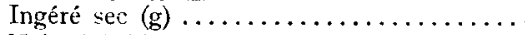 & 915,6 & 917,5 \\
\hline$N$ ingéré $(g) \ldots \ldots \ldots \ldots \ldots \ldots \ldots \ldots \ldots$ & 26,63 & 40,71 \\
\hline \multicolumn{3}{|l|}{ Composition corporell: : } \\
\hline Protéines p. $100 \ldots \ldots$ & 18,95 & 20,62 \\
\hline Lipides p. $100 \ldots \ldots \ldots \ldots \ldots \ldots \ldots \ldots$ & 18,14 & 13,67 \\
\hline
\end{tabular}

L'examen des résultats de composition corporelle fait ressortir une grande similitude d'un lot à l'autre pour les animaux de même sexe ; ceci est vrai aussi bien chez les animaux qui ont présenté une même vitesse de croissance et dont le poids final est comparable (lot au poisson et lot à l'arachide) que chez ceux pour qui ces critères ont été différents (lot au gluten comparé aux deux autres). I1 est, en outre, à noter que, compte tenu de ces critères, les animaux sont beaucoup plus maigres que ceux obtenus dans d'autres expériences à l'aide de régimes mixtes contenant les mêmes protéines (RÉRAT et al., résultats non publiés). Ainsi, dans le tableau Io, nous avons rapporté les résultats moyens de deux lots d'animaux, d'une part le lot recevant les protéines de poisson et le régime énergétique à volonté dans l'expérience présente, d'autre part un lot ingérant un régime mixte à 16 p. Ioo des mêmes protéines. La vitesse de croissance et le poids final sont comparables, mais les animaux en régime mixte présentent des carcasses moins riches en protéines et plus grasses 
que ceux qui sont en régime séparé. La consommation d'énergie est la même dans les deux cas, mais la consommation de protéines est beaucoup plus élevée en libre choix. I1 semble donc que les animaux qui ont le libre choix de l'azote et de l'énergie soient plus maigres qu'en régime complet, $d u$ fait qu'ils ingèrent plus d'azote. Cependant, les deux lots d'animaux n'ayant pas été traités simultanément, cette hypothèse nécessite une vérification.

En définitive, lorsque le rat reçoit, à volonté, dans deux mangeoires séparées, des matières azotées et des éléments énergétiques, il constitue des régimes qui renferment un taux élevé de protéines (autour de $30 \mathrm{p}$. I00) quelle qu'en soit la valeur biologique. Dans tous les cas, il ajuste sa consommation totale d'énergie en fonction de son poids, mais il ne parvient pas à faire la part des calories protéiques et des calories non protéiques. Comme il a déjà été démontré par ailleurs (HENRY et RERAT, I962), cet ajustement se produit également lorsqu'on limite l'apport azoté, que ce soit en quantité ou en qualité. On peut donc conclure que le rat, quelles que soient les conditions d'alimentation azotée et dans les limites de nos expériences, se fixe un niveau d'alimentation énergétique qui est fonction de l'impulsion de croissance permise par les protéines.

\section{Reçu pour publication en février 1963.}

\section{SUMMARY}

THE VOLUNTARY INTAKE OF ENERGY AND PROTEIN OF THE GROWING RAT AS STUDIED BY THE SELF SELECTION METHOD

The voluntary intake of energy and protein of the growing rat was measured by feeding free choice during 8 weeks, in two separate troughs, a protein diet and a protein-free diet, the two $a d$ libitum. Three lots of 9 rats were compared, using one of the following protein sources : fish meal, peanut oil meal and wheat gluten.

The results indicate that the rat is not able to adjust its protein consumption to the level of its need. But the voluntary energy consumption is regulated through the body weight : in a given body weight range $(70$ to $35 \circ \mathrm{g}$ ) the daily calorie intake varies as the body weight raised to the same power $(0,32$ to 0,35$)$ whatever the quality of the protein be fed. It appears that the growing rat is able to regulate its daily food consumption as a whole according to its growth impulse, but it does not make the right selection of the protein.

\section{RÉFÉRENCES BIBLIOGRAPHIQUES}

Abraham J., Calet C., Rerat A., Jacquot R., ig6r. Solidarité des besoins énergétique et protéique de croissance : l'ajustement spontané des calories et des protéines. C. R. Acad. Sci. Paris, 253, 2768-2770.

Ashoenasy-Lelu P., i951. L'ingestion alimentaire en fonction de la composition du régime. Application à la régulation du besoin azoté chez le Rat. Ann. Nutr. Paris, 5, 453-483.

Atwater W. O., Bryant A. P., I903. The chemical composition of american food materials. $U . S$. Dept. Agr. Bull., 28.

Bligh E. G., Dyer W. J., 1959. A rapid method of total lipid extraction and purification. Canad. J. Biochem. Physiol., 37, 91 I-917.

Brody S., 1945. Bioenergetics and groweth, ro23 pp. Reinhold Publ. Corp., New York.

Calet C., Jouandet C., Baratou J., I96r. Variation de la consommation spontanée d'énergie du poussin en fonction de la nature des matières azotées du régime. Ann. Biol. anim. Bioch. Biophys., 1, I-9. 
CowglLL G. R., I928. The energy factor in relation to food intake : experiments on the dog. Amer. J. Physiol., 85, 45-64.

Evvard J. M., I9I5. Is the appetite of swine a reliable indication of physiological needs? Proc. Iowa Acall. Sci., 22, 375-409.

Hegsted D. M., Haffenreffer V. R., 1949. Calorie intake in relation to the quantity and quality of protein in the diet. Amer. J. Physiol., 15\%, r41-148.

HENRY Y., RERAT A., ig62. Influence de la qualité et de la quantité des matières azotées ingérées sur la consommation spontanée d'énergie chez le rat en croissance. Ann. Biol. anim. Bioch. Biophys., 2, 267-276.

Kon S. K., 193r. The self-selection of food constituents by the Rat. Biochem. J., 25, 473-48r.

Lassiter J. W., Terrill S. W., Becker D. E., Norton H. W., i955. Protein levels for pigs as studied by growth and self-selection. J. Anim. Sci., 14, 482-491.

Mitchell H. H., Block R. J., 1946. Some relationships between the aminoacid contents of proteins and their nutritive value for the rat. J. Biol. Chem., 163, 599-620.

Mitchell H. S., Mendel L. B., I92r. Studies in nutrition. The choice between adequate and inadequate diet, as made by rats and mice. Amer. J. Physiol., 58, 2 I I-225.

Munro H. N., i95. Carbohydrate and fat as factors in protein utilization and metabolism. Physiol. Rev., 31, 449-488.

Osborne T. B., MENDEL L. B., I9I7. The relative value of certain proteins and protein concentrates as supplements to corn gluten. J. Biol. Chem., 29, 69-92.

Osborne T. B., Mendei L. B., igr8. The choice between adequate and inadequate diets, as made by rats. J. Biol. Chem., 35, r9-27.

PION R., 1963. (Communication personnelle).

Rerat A., Henry Y., Fevrier C., Lougnon J., ig63. (Résultats non publiés).

RERAT A., HENRY Y., JACQLOT R., 1963. Relation entre la consommation spontanée d'énergie et la rétention azotée chez le rat en croissance. C. R. Acad. Sci. Paris, 256, 787-789.

Riciter C. P., Holt L. E. jr, Barelare B. jr., 1938. Nutritional requirements for normal growth and reproduction in rats studied by the self-selection method. Amer. J. Physiol., 122, 734-744.

Rubner M., 1902. Die Gesetze des Energieverbrauchs be? der Ernährung, Deutiche, Leipzig und Wien.

SNEDECOR G. W., 1956. Statistical methods applied to experiments in agriculture and biology. $5^{\mathrm{e}}$ ed., $534 \mathrm{pp}$. The Iowa State University Press, Ames, Iowa. 